\title{
A controlled assessment of an asthma self-management plan involving a budesonide dose regimen
}

\author{
J.G. Ayres*, L.M. Campbell**, R.M.A. Follows ${ }^{+}$on behalf of the \\ OPTIONS research group
}

A controlled assessment of an asthma self-management plan involving a budesonide dose regimen. J.G. Ayres, L.M. Campbell, R.M.A. Follows on behalf of the OPTIONS research group. CERS Journals Ltd 1996.

ABSTRACT: Our aim was to assess the efficacy of budesonide (Pulmicort ${ }^{\circledR}$ Turbohaler ${ }^{\circledR}$, Astra) used as part of a self-management plan in a group of patients with chronic asthma.

One hundred and twenty five patients with nocturnal asthma symptoms, despite the use of inhaled prophylactic and $\beta_{2}$-agonist therapy, were randomized to inhaled budesonide 200,400 or $800 \mu \mathrm{g}$ b.i.d. either with dose adjustments made by the physician, i.e. doctor-managed (DM; $n=64)$, or as part of a self-management plan (SM; $\mathrm{n}=61$ ). The SM group were allowed to adjust their dose according to written guidelines based on morning peak flow.

At the end of the 6 month treatment period, there were no significant differences detected between the DM and the SM groups either from the clinic or diary card data. Both groups demonstrated a significant reduction in the number of sleep-disturbed nights, by $75 \%$ in the DM group and $77 \%$ in the SM group, at the end of the study.

In conclusion, for patients with mild-to-moderate asthma, either a doctor-adjusted dose regimen or a peak flow based self-management plan involving budesonide is equally efficacious. For some patients, a simple regimen, adjusted by the physician at clinic visits, may be easier to follow.

Eur Respir J., 1996, 9, 886-892.
* Chest Research Institute, Birmingham Heartlands Hospital, Birmingham, UK. **Southbank Surgery, Kirkintilloch, Scotland. +Clinical Research Dept, Astra Pharmaceuticals Ltd, Kings Langley, UK.

Correspondence: J.G. Ayres

Dept of Respiratory Medicine

Birmingham Heartlands Hospital

Bordesley Green East

Birmingham

B9 5SS

UK

Keywords: Asthma

budesonide

self-management

Turbohaler ${ }^{\circledR}$

Received: March 131995

Accepted after revision December 61995

This study was sponsored by Astra Pharmaceuticals Ltd, UK.
Regular inhaled corticosteroid therapy is well-established as prophylactic treatment for asthma $[1,2]$. Asthma is characteristically a variable disease; thus, patients prescribed a fixed daily dose of inhaled steroid may not always receive an appropriate dose during periods of poor asthma control, when the daily dose of inhaled steroid may need to be temporarily increased, or of good asthma control, with no or few symptoms over several weeks, when the dose may be stepped down.

The use of peak flow or symptom-based management plans has been recommended in the British Thoracic Society Guidelines [1], allowing patients the ability to control their own asthma management with less recourse to their doctor. Studies of peak flow based management plans have shown significant reductions in the amount of medication used [3] in asthma severity, nights woken and an increase in baseline lung function [4]. In patients with milder asthma, as seen in general practice, symptom only management plans have shown similar benefits [5], and may be the preferable method in such patients. It is possible, however, that the decreased asthma morbidity seen in these studies was the result of regular supervision rather than the introduction of a self-management plan.
Conversely the Grampian Asthma Study of Integrated Care (GRASSIC) from Aberdeen, involving self-management based on peak flow, found no significant differences between patients randomized either to self-management or conventional monitoring, after one year [6]. Intriguingly, the self-management group showed a trend towards visiting their general practitioner more frequently. Furthermore, the more severe patients in the self-management group used more than twice as many courses of oral steroids [6].

Although self-management appears attractive, its success depends on the effort put into training by the doctor and the capability and motivation of the patient. A simple dose regimen adjusted by the physician at clinic visits, however, is easy for the patient and requires less input from the doctor. If adequate treatment and regular supervision are the major prerequisites for controlled asthma then a simple dose regimen could be the better option for some patients unable to cope with self-management plans.

The aim of this study was to assess the efficacy and acceptability of an asthma self-management plan, based on regular peak expiratory flow (PEF) measurements compared to a standard dose regimen involving budesonide via Turbohaler®. 


\section{Methods}

\section{Patients}

Patients with a history of chronic asthma were studied. Each patient was aged $17 \mathrm{yrs}$ or older, with a documented increase $(\geq 15 \%)$ in forced expiratory volume in one second (FEV1) following inhalation of a $\beta_{2}$-agonist and/or documented diurnal variation in PEF $(\geq 15 \%)$ plus at least one documented exacerbation of their asthma in the previous 6 months which required contact with a doctor/nurse. Patients had disturbed sleep (which included early morning awakening due to asthma) on at least 3 nights in the week prior to enrolment into the study despite using inhaled prophylactic therapy (inhaled corticosteroids $400-1,600 \mu \mathrm{g} \cdot \mathrm{day}^{-1}$, sodium cromoglycate or nedocromil sodium) for a minimum of 3 months.

Patients were excluded for the following reasons: use of long-acting inhaled $\beta_{2}$-agonists, anticholinergics, corticosteroids (other than by the inhaled route) within the past 4 weeks; routine/regular use of a Turbohaler ${ }^{\circledR}$ in the 6 months before entry; respiratory tract infection at, or within 2 weeks of entry; significant disease that could have interfered with the study; pregnancy, lactation or lack of adequate contraception; and previous participation in the study or participation in any other clinical study in the 6 months before entry. All patients gave written informed consent, and the study was approved by appropriate independent Ethics Committees for each participating centre.

\section{Study design}

The study was an open, randomized, parallel group trial of $24 \pm 2$ weeks duration. After randomization, patients made an additional four visits to the clinic at $6 \pm 1$ weekly intervals. Patients were randomized at entry to receive budesonide (Turbohaler® 200; Astra: 200, 400 or $800 \mu \mathrm{g} \mathrm{b.d.)} \mathrm{either} \mathrm{as} \mathrm{part} \mathrm{of} \mathrm{a} \mathrm{self-management} \mathrm{(SM)}$ plan or as a doctor-managed (DM) regimen. All patients received terbutaline (Bricanyl ${ }^{\circledR}$ Turbohaler@; Astra: 500 ug p.r.n.); oral steroids and nebulized $\beta_{2}$-agonists could be prescribed (based on clinical need) as rescue medication.

At randomization, the investigator determined the appropriate starting dose of budesonide based on the patient's current peak flow. The potential normal PEF was calculated for each patient at entry as either the predicted PEF [7] or the highest documented PEF, if available. If a patient had taken a course of oral steroids (20-60 $\mathrm{mg} \cdot \mathrm{day}^{-1}$ for over 1 week) within the previous year, the potential normal PEF was defined as the highest documented PEF at the end of that course of treatment.

Doctor-managed regimen. Patients randomized to receive the doctor-managed dose regimen had their budesonide dose adjusted by the investigator only, over the range 200-800 ug b.d., when they attended the clinic visits. The investigator adjusted the dose according to the diary card data for asthma control over the previous treatment period.

Self-management regimen. Patients in the self-management group were able to adjust their own budesonide dose according to written guidelines based on morning PEF measurements (best of three attempts before terbutaline use) as a percentage of their "normal" PEF.

There were three dose levels based on morning PEF as a percentage of the potential normal PEF: $>85 \%$ of the potential normal PEF (200 $\mu \mathrm{g}$ b.d.); $70-85 \%$ of the potential normal PEF $(400 \mu \mathrm{g}$ b.d.); and $<70 \%$ of the potential normal PEF ( $800 \mu \mathrm{g}$ b.d.). This dose could subsequently be increased or decreased if morning PEF changed.

Each patient was directed to contact the investigator if their PEF fell below $60 \%$ of their potential normal PEF at any time during the study or if they felt that their asthma symptoms were not adequately controlled. The investigator could then either change the dose of concurrent antiasthma medication, introduce nebulized $\beta_{2}-$ agonists, or initiate oral corticosteroid treatment (30-60 $\mathrm{mg} \cdot \mathrm{day}^{-1}$ ) for as many days as necessary to raise morning PEF to $>85 \%$ of normal for 2 consecutive days, or withdraw the patient from the study.

Criteria for decreasing the daily budesonide dose were as follows:

1. Morning PEF increased from $70-85 \%$ to $>85 \%$ of the potential normal PEF for 5 consecutive days; dose halved to $200 \mu \mathrm{g}$ b.d.

2. Morning PEF increased from $<70 \%$ to $70-85 \%$ of the potential normal PEF for 5 consecutive days; dose halved to $400 \mu \mathrm{g} b . d$.

3. Morning PEF increased from $<70 \%$ to $>85 \%$ of the potential normal PEF in less than 5 days; dose halved to $400 \mu \mathrm{g} \mathrm{b.d}$. after 5 consecutive days $>70 \%$ of potential normal PEF; dose halved to $200 \mu \mathrm{g} \mathrm{b.d}$. if morning $\mathrm{PEF}$ remained $>85 \%$ of the potential normal PEF for 5 more consecutive days.

Criteria for increasing the daily budesonide dose were as follows:

1. Morning PEF fell from $70-85 \%$ to $<70 \%$ of the potential normal PEF for 2 consecutive days; dose doubled to $800 \mu \mathrm{g}$ b.d.

2. Morning PEF fell from $>85 \%$ to $70-85 \%$ of the potential normal PEF for 2 consecutive days; dose doubled to $400 \mu \mathrm{g} b . d$.

3. Morning PEF fell from $>85 \%$ to $<70 \%$ of the potential normal PEF for (or over) 2 consecutive days; dose increased to $800 \mu \mathrm{g}$ b.d.

\section{Clinic assessments}

Lung function was assessed at each clinic visit by measurements of FEV1, forced vital capacity (FVC) and PEF. Hospital centres used their own spirometers for measuring $\mathrm{FEV} 1$ and $\mathrm{FVC}$, whereas general practice centres were provided with a handheld spirometer (Micro-Medical). PEF was always measured using a mini-Wright peak flow meter. Patients were asked to try not to use terbutaline during the $5 \mathrm{~h}$ before each visit. 
At entry, the investigator recorded the number of sleepdisturbed nights (including early morning awakening) due to asthma during the previous week recalled by the patient. At each visit, the investigator graded the overall severity of asthma and the severity of individual symptoms (cough at rest, cough on activity, wheeze at rest, wheeze on activity, difficulty in breathing, interference with daily life, sleep disturbance) using the following scale: $0=$ none; $1=$ mild; $2=$ moderate; and $3=$ severe.

At entry, patients were asked whether they liked doing, or needed to do, 10 activities (gardening; entering smoky environments; going to school/college/work; getting a specific job/promotion; housework/do-it-yourself (DIY); sports/physical exercise (PE)/keep-fit; going out walking/shopping; being with pets/animals; going out in cold weather; going out with friends), and whether their ability to do each activity was limited by asthma. At each subsequent visit, patients were asked whether they could perform each activity better than at entry. In addition, an activity was selected at entry as a personal target for improvement, and at each subsequent visit patients were asked to assess whether their ability to perform the activity was much improved, improved, the same or worse than at entry.

An overall assessment of treatment both by the investigator and patient was performed on completion of the study.

Adverse events were recorded in response to open questions at each visit or on withdrawal from the study. An oropharyngeal inspection was performed at each visit to detect clinical evidence of oral candidiasis.

\section{Patient diary cards}

Diary cards were completed each morning (on rising) and each evening (at bedtime): morning and evening PEF (best of three blows before bronchodilator use), and the number of daytime and nighttime terbutaline and budesonide inhalations were recorded. Presence or absence of sleep disturbance due to asthma was recorded. In the evening, patients graded the severity of their asthma symptoms (cough, wheeze, difficulty in breathing) on a scale of $0-3(0=$ none; $1=$ mild; $2=$ moderate; $3=$ severe $)$ experienced during the day.

\section{Peak flow charts}

Patients in the self-management group were provided with peak flow charts and peak flow meters were marked with colour coded lines highlighting the values corresponding to $85 \%$ (green), $70 \%$ (amber) and $60 \%$ (red) of a patient's potential normal PEF. Written guidelines for dose changing as well as examples were provided with each peak flow chart.

\section{Statistical analyses}

The primary outcome variable was the number of sleepdisturbed nights due to asthma. Secondary variables included lung function, asthma symptom scores and activity assessments. Analysis, including all available data, was performed on all assessments and a per-protocol (PP) analysis was performed on the number of sleep-disturbed nights due to asthma and the use of study medication. It was planned to randomize 130 patients into the study, aiming for 52 per group completing the study. This would provide $80 \%$ power at the $5 \%$ significance level to detect a $28 \%$ reduction in the number of sleep-disturbed nights due to asthma.

The mean number of sleep-disturbed nights for the week before entry and the last week of treatment were compared within and between groups using the Wilcoxon signed rank test and the Wilcoxon rank sum test, respectively. Mean symptom severities at entry and at the end of treatment were also analysed within and between groups using the Wilcoxon signed rank test and the Wilcoxon rank sum test, respectively. Analyses of the ability to perform target activities at the end of treatment (much improved/improved, the same as or worse than at entry) were compared within and between groups using a sign test and a Chi-squared test, respectively. Overall assessment questions were compared using a Chisquared test. Mean lung function measurements at entry and at the end of treatment were analysed within and between groups using a paired t-test and a two-sample t-test, respectively. For diary card PEF and symptoms, only the last 14 days of available data before the end of treatment were used. Between group comparisons were performed using a two-sample t-test for PEF data and the Wilcoxon rank sum test for symptoms. All diary card data were used to calculate the mean daily budesonide and terbutaline doses. Between group comparisons were performed using the Wilcoxon rank sum test. Data are presented as mean \pm SEM, unless otherwise stated.

\section{Results}

\section{Patients}

One hundred and twenty six patients were randomized into the study. One hundred and twenty five patients received treatment and were included in the analysis. Fifty patients completed the study without violating the protocol and were included in the per-protocol analysis. Demographic characteristics of patients for both analyses are presented in table 1 . Thirty two patients discontinued treatment and were withdrawn from the study, 22 in the SM group and 10 in the DM group. Patients were withdrawn for the following reasons: noncompliance (SM 8; DM 4); asthma deterioration (SM 3; DM 3); pregnancy/lack of contraception (SM 2); excluded medication (SM 2); adverse events (SM 5; DM 1); and other (SM 2; DM 2).

\section{Sleep disturbance}

There were significant $(\mathrm{p}<0.001)$ reductions in disturbed sleep of $3.89 \pm 0.44$ nights.week ${ }^{-1}(77 \%)$ for SM patients and $3.87 \pm 0.36$ nights.week ${ }^{-1}(75 \%)$ for DM patients (fig. 1) ( $\mathrm{p}=0.95$ between groups). Sleep disturbance scores fell significantly $(\mathrm{p}<0.001)$ for SM patients 
Table 1. - Characteristics at entry for patients included in the analyses

\begin{tabular}{|c|c|c|c|c|}
\hline \multirow[b]{2}{*}{ Variable } & \multicolumn{2}{|c|}{ Full analysis } & \multicolumn{2}{|c|}{ Per-protocol } \\
\hline & SM & DM & SM & DM \\
\hline Patients $\mathrm{n}$ & 61 & 64 & 21 & 29 \\
\hline Age yrs & $44 \pm 2$ & $47 \pm 2$ & $42 \pm 3$ & $50 \pm 3$ \\
\hline Height $\mathrm{cm}$ & $166 \pm 1.2$ & $166 \pm 1.0$ & $166 \pm 2.0$ & $166 \pm 1.5$ \\
\hline Weight $\mathrm{kg}$ & $72 \pm 2$ & $73 \pm 2$ & $70 \pm 3$ & $72 \pm 3$ \\
\hline $\operatorname{Sex} \mathrm{M} / \mathrm{F}$ & $23 / 38$ & $28 / 36$ & $7 / 14$ & $13 / 16$ \\
\hline$\%$ potential normal PEF & $79 \pm 2$ & $72 \pm 2$ & $79 \pm 4$ & $71 \pm 3$ \\
\hline (on prophylaxis)* & $32-107$ & $33-119$ & $40-105$ & $33-96$ \\
\hline $\begin{array}{l}\text { Inhaled steroid dose } \\
\mu \mathrm{g} \cdot \text { day }^{-1}\end{array}$ & $\begin{array}{r}649 \pm 51 \\
(\mathrm{n}=60)\end{array}$ & $\begin{array}{r}717 \pm 63 \\
(n=63)\end{array}$ & $\begin{array}{r}624 \pm 72 \\
(n=21)\end{array}$ & $\begin{array}{r}724 \pm 66 \\
(\mathrm{n}=28)\end{array}$ \\
\hline \multicolumn{5}{|l|}{ Budesonide starting dose } \\
\hline Mean $\mu g \cdot$ day $^{-1}$ & $892 \pm 61$ & $981 \pm 56$ & $857 \pm 101$ & $966 \pm 85$ \\
\hline $400 \mu \mathrm{g} \cdot \mathrm{day}^{-1} \mathrm{n}$ & 20 & 11 & 7 & 6 \\
\hline $800 \mu \mathrm{g} \cdot$ day $^{-1} \mathrm{n}$ & 24 & 33 & 9 & 14 \\
\hline $1,600 \mu \mathrm{g} \cdot$ day $^{-1} \mathrm{n}$ & 17 & 20 & 5 & 9 \\
\hline $\begin{array}{l}\text { Duration of asthma } \\
\text { treatment yrs }\end{array}$ & $10.1 \pm 1.4$ & $13.0 \pm 1.8$ & $12.7 \pm 3.0$ & $14.3 \pm 3.2$ \\
\hline
\end{tabular}

Data are presented as the number of patients or the mean \pm SEM. *: mean \pm SEM, and range in parenthesis. M: male: F: female; DM: doctor-managed; SM: self-management; PEF: peak expiratory flow.

from $1.79 \pm 0.09$ to $0.67 \pm 0.14$ (63\% reduction), and for DM patients from $1.89 \pm 0.10$ to $0.69 \pm 0.13$ (63\% reduction).

Similar results were found in the per-protocol analysis, with significant reductions in the number of sleepdisturbed nights due to asthma. For the SM group, the number of sleep-disturbed nights decreased by $3.71 \pm 0.63$ nights.week ${ }^{-1}(74 \%)$, and for the DM group by $3.80 \pm 0.50$ nights $\cdot$ week $^{-1}(75 \%)(\mathrm{p}=0.96$ between groups $)$.

\section{Daytime symptoms}

Overall severity of asthma, wheeze, wheeze on activity and difficulty in breathing recorded at the clinic

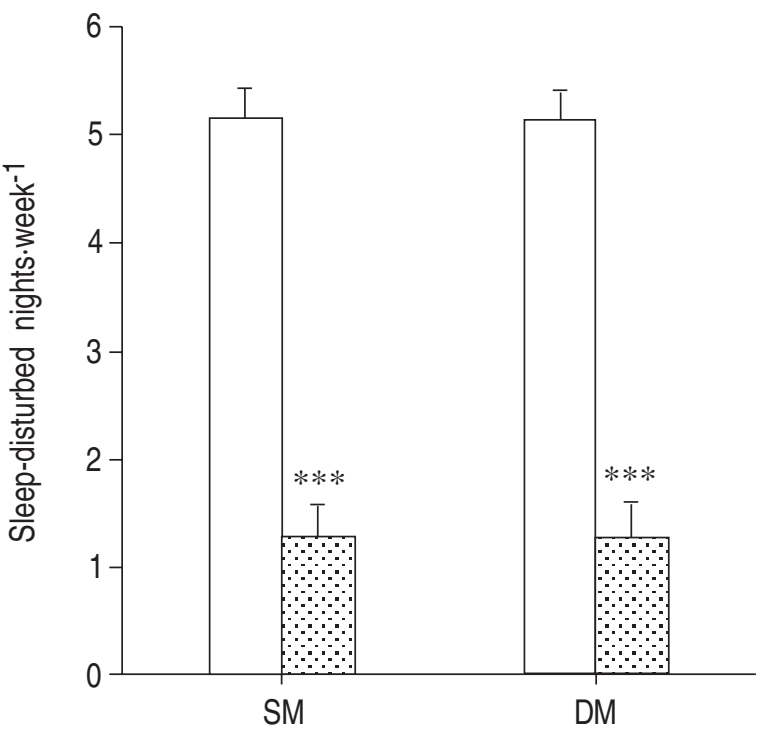

Fig. 1. - Number of sleep-disturbed nights due to asthma during the week before entry and the last week of treatment. Values are presented as mean \pm SEM. $\square$ : week before entry; $\square$ : last week of budesonide treatment. SM: self management; DM: doctor managed; ***: $\mathrm{p}<0.001$, within group comparisons. Between group comparison, $\mathrm{p}=0.96$. visits, were significantly improved both for SM and DM groups (table 2), with improvement in cough, both at rest and on activity, being significant for the DM group only. Diary card data did not reveal any significant differences between treatments for cough, wheeze and shortness of breath. As no baseline diary card data were recorded, within group comparisons were not possible.

\section{Activity scores}

The degree to which asthma interfered with patients' daily lives improved significantly from entry to the end of treatment. Overall scores fell for the SM group from $1.46 \pm 0.12$ to $0.87 \pm 0.14(\mathrm{p}<0.01)$ and for the $\mathrm{DM}$ group from $1.48 \pm 0.12$ to $0.85 \pm 0.13(\mathrm{p}<0.001)$. The difference between groups was not significant.

Both groups reported significant $(\mathrm{p}<0.001)$ improvements in their ability to perform their target activity (fig. 2 ), but between group differences were not significant $(\mathrm{p}=0.35)$.

\section{Lung function}

Clinic visit prebronchodilator lung function measurements for SM patients and DM patients did not increase significantly, and the differences between groups were also nonsignificant (table 3 ).

At the end of treatment, there were no significant between group differences in diary card morning or evening peak flows (SM versus DM): morning PEF (382 \pm 16 versus $370 \pm 17 \mathrm{~L} \cdot \mathrm{min}^{-1} ; \mathrm{p}=0.62$ ), evening PEF ( $395 \pm 17$ versus $377 \pm 17 \mathrm{~L} \cdot \mathrm{min}^{-1} ; \mathrm{p}=0.48$ ).

\section{Medication usage}

Analysis of budesonide use (mean dose \pm SD) throughout the study for SM patients $\left(926 \pm 56 \mu \mathrm{g} \cdot\right.$ day $\left.^{-1}\right)$ and DM patients $\left(970 \pm 52 \mu \mathrm{g} \cdot\right.$ day $\left.^{-1}\right)$ did not demonstrate a 
Table 2. - Asthma symptom severity scored (0-3) at the clinic visits at the beginning and end of treatment for both treatment groups

\begin{tabular}{|c|c|c|c|c|c|c|c|c|}
\hline & \multicolumn{4}{|c|}{ Self-management } & \multicolumn{4}{|c|}{ Doctor-managed } \\
\hline & Entry & $\begin{array}{l}\text { End of } \\
\text { treatment }\end{array}$ & $\begin{array}{c}\text { Decrease } \\
\%\end{array}$ & p-value & Entry & $\begin{array}{l}\text { End of } \\
\text { treatment }\end{array}$ & $\begin{array}{c}\text { Decrease } \\
\%\end{array}$ & p-value \\
\hline $\begin{array}{l}\text { Overall severity } \\
\text { of asthma }\end{array}$ & $1.77 \pm 0.08$ & $1.38 \pm 0.12$ & 22 & $<0.01$ & $1.91 \pm 0.07$ & $1.39 \pm 1.11$ & 27 & $<0.001$ \\
\hline Cough at rest & $1.00 \pm 0.11$ & $0.87 \pm 0.15$ & 13 & 0.65 & $1.08 \pm 0.12$ & $0.69 \pm 0.10$ & 36 & $<0.01$ \\
\hline Cough on activity & $1.48 \pm 0.12$ & $1.28 \pm 0.17$ & 14 & 0.24 & $1.75 \pm 0.12$ & $1.30 \pm 0.14$ & 26 & $<0.01$ \\
\hline Wheeze at rest & $0.97 \pm 0.11$ & $0.74 \pm 0.13$ & 24 & $<0.05$ & $1.25 \pm 0.13$ & $0.67 \pm 0.10$ & 46 & $<0.001$ \\
\hline Wheeze on activity & $1.74 \pm 0.11$ & $1.38 \pm 0.15$ & 21 & $<0.01$ & $1.94 \pm 0.11$ & $1.30 \pm 0.12$ & 33 & $<0.001$ \\
\hline $\begin{array}{l}\text { Difficulty in } \\
\text { breathing }\end{array}$ & $1.41 \pm 0.12$ & $0.85 \pm 0.14$ & 40 & $<0.01$ & $1.47 \pm 0.12$ & $0.96 \pm 0.14$ & 35 & $<0.01$ \\
\hline
\end{tabular}

Values are presented as mean \pm SD.

significant difference $(\mathrm{p}=0.72)$. The per-protocol analysis demonstrated that DM patients used $15 \%$ more budesonide $\left(980 \pm 80 \mu \mathrm{g} \cdot \mathrm{day}^{-1}\right)$ than SM patients $(850 \pm 90$ $\left.\mu \mathrm{g} \cdot \mathrm{day}^{-1}\right)$, although this difference was not significant $(\mathrm{p}=0.23)$.

Similarly, analysis of terbutaline use throughout the study did not demonstrate a significant difference between groups.

\section{Overall assessment}

Investigator assessment of the benefit to the patient of each form of management showed equivalent results

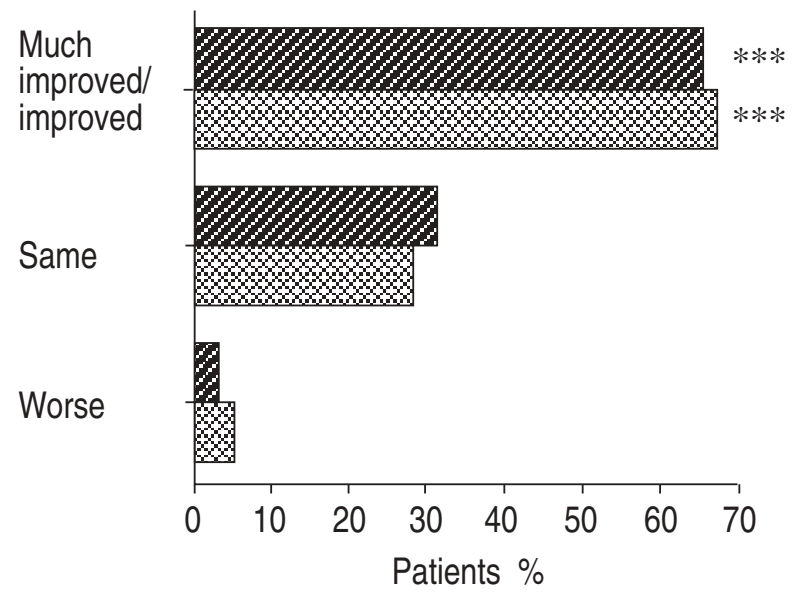

Fig. 2. - Proportion of patients whose ability to perform their target lifestyle activity at the end of treatment was much improved/improved, the same, or worse than at entry. $\square$ :doctor-managed; $\square$ : selfmanagement. $* * *: \mathrm{p}<0.001$, within group comparisons. Between group comparison, $\mathrm{p}=0.35$. both for SM and DM (87\% SM, 78\% DM; p=0.29) with similar results for the patients' own assessment (92\% SM, 77\% DM; $p=0.09)$. Eighty seven percent of patients preferred self-management to their previous management system, and $87 \%$ expressed a wish to continue using the self-management plan.

\section{Adverse events}

A total of $88(70 \%)$ patients (42 (69\%) SM, 46 (72\%) DM), reported 215 adverse events (table 4). There were no differences between the two groups. With the exception of oral candidiasis and tremor/palpitations, none of the adverse events were thought to be attributable to the study medication. Six patients discontinued treatment as a result of adverse events: five in the SM group (oral candidiasis; burning leg pains and hot flushes; systemic viral illness; palpitations; nausea and headache) and one in the DM group (tremor and palpitations).

Serious adverse events were reported by three SM patients (two hospitalized due to an asthma exacerbation, and one hospitalized with cervical spondylosis), and one DM patient who had two transient ischaemic attacks.

\section{Discussion}

The $77 \%$ decrease in the number of sleep-disturbed nights reported by the SM group in this study is comparable to the $84 \%$ decrease demonstrated by BEASLEY et al. [4] (1989) using a similar self-management plan. The DM group, however, also demonstrated a decrease of $75 \%$, and the reductions in severity for the majority

Table 3. - Prebronchodilator clinic visit lung function recorded at the initial and final clinic visits

\begin{tabular}{lccccccc}
\hline & \multicolumn{3}{c}{ Self-management } & & \multicolumn{3}{c}{ Doctor-managed } \\
\cline { 2 - 4 } \cline { 5 - 7 } & Entry & $\begin{array}{c}\text { End of } \\
\text { treatment }\end{array}$ & p-value & & Entry & $\begin{array}{c}\text { End of } \\
\text { treatment }\end{array}$ & p-value \\
\hline PEF L-min & $395 \pm 13$ & $405 \pm 18$ & 0.12 & & $365 \pm 14$ & $393 \pm 18$ & 0.06 \\
FEV1 L & $2.3 \pm 0.1$ & $2.3 \pm 0.2$ & 0.64 & & $2.0 \pm 0.1$ & $2.2 \pm 0.1$ & 0.11 \\
FVC L & $3.1 \pm 0.1$ & $3.1 \pm 0.2$ & 0.62 & & $2.8 \pm 0.1$ & $2.9 \pm 0.1$ & 0.41 \\
\hline
\end{tabular}

Values are presented as mean \pm SD. PEF: peak expiratory flow; FEV1: forced expiratory volume in one second; FVC: forced vital capacity. 
Table 4. - Adverse effects, separated by treatment and body system

\begin{tabular}{lrrc}
\hline & SM & DM & Total \\
\hline Respiratory & 41 & 51 & 92 \\
Gastrointestinal & 6 & 13 & 19 \\
Central nervous & 19 & 11 & 30 \\
Musculoskeletal & 16 & 18 & 34 \\
Skin & 5 & 4 & 9 \\
Cardiovascular & 2 & 6 & 8 \\
Other & 7 & 16 & 23 \\
\hline Total & 96 & 119 & 215 \\
\hline
\end{tabular}

SM: self-management; DM: doctor-managed.

of asthma symptoms were greater, albeit not significantly, for this group. Moreover, a previous trial in an equivalent patient population reported that patients given a fixed dose of $800 \mu \mathrm{g}$ daily budesonide via Turbohaler® had their sleep disturbed on only 1.26 nights.week ${ }^{-1}$ [8], which is comparable to the 1.14 nights $\cdot$ week $^{-1}$ shown in both groups in this study.

One conclusion that could be drawn from this study is that peak flow based self-management plans per se do not reduce asthma morbidity, compared to regular supervision and monitoring combined with appropriate treatment. Similar doubts regarding the suitability of peak flow management plans for unselected asthmatics have been raised by other studies [5, 6]. For patients with less severe asthma, a simple dose regimen adjusted by a physician or nurse combined with regular review of the patient's progress may be easier for the patient to follow and may result in a similar level of asthma control. It could equally be argued that it is just this group which need not be seen regularly by their physicians, who should be in control of their own asthma, although a decision as to which method of management is appropriate is, in the end, best left to the physician to decide.

Determining the appropriate dose of inhaled corticosteroid therapy requires titration of the dose until symptom control is achieved. Patients who have previously been well-controlled but begin to show signs of asthma deterioration (e.g. nocturnal symptoms and increased $\beta_{2^{-}}$ agonist use) usually respond to having their anti-inflammatory medication stepped up (often doubled) to reduce airway inflammation and bring the symptoms back under control, according to treatment guidelines [1]. Similarly, the guidelines emphasize the importance of stepping down treatment during periods of good control, if the patient has had no or few symptoms for some time. For some patients self-management will be suitable whilst others need the supervision of a nurse or doctor.

The self-management plan used in this trial was based on those described in previous trials [4, 5]. However, three inhaled steroid dose levels were available, all of which are frequently used in normal practice, and patients were not permitted to initiate oral steroid treatment without contacting the investigator. In view of the similarity of the peak flow based self-management plans issued to the patients, it may not be surprising that patients in these trials benefited from similar improvements in asthma morbidity.
At entry, all patients in the study were symptomatic despite using inhaled prophylactic medication, probably indicating that the dose was too small and/or was not being taken effectively, or that it was not being taken at all. All patients received budesonide via Turbohaler ${ }^{\circledR}$ during the trial, and the mean inhaled steroid dose for both groups was higher at randomization than at entry, so the mere adjustment of the inhaled steroid dose at randomization may have been sufficient to control their asthma, and thus mask any possible benefit of using a management plan. There were, however, no significant differences between the groups at entry in terms of symptom scores or peak flow.

Despite investigators being motivated and all patients being considered capable of self-management at entry, $15 \%$ of those randomized to the self-management group were withdrawn due to noncompliance compared to $6 \%$ of the doctor-managed group. Although minor infringements of the self-management plan were common for the 39 patients that completed the study, $26 \%$ took the incorrect dose on more than $25 \%$ of days. Patients who failed to follow the self-management plan correctly may have found it too complicated, whilst for others it may have been too inflexible. In view of the time and effort required to introduce patients to self-management, it may be wiser to be more selective and to tailor self-management plans to the needs of each patient, taking into account their ability and motivation. Individualized plans may encourage greater compliance and increase the chance of more appropriate inhaled steroid use. Removal of all protocol violators from the analysis, however, still demonstrated large but almost identical decreases in the number of sleep-disturbed nights for both groups. Although the trend for lower inhaled steroid use by the self-management group may indicate more appropriate use, this did not reach a statistically significant level.

In conclusion, this study showed that symptomatic adult asthmatics benefit equally by the introduction of budesonide Turbohaler® treatment either as part of a self-management plan or dose regimen altered by the physician with regular supervision. Which system suits which patient will be a matter of personal decision by the patient and physician together.

Acknowledgements: The following centres contributed to this study: J.R.M. Lough (Airdrie Health Centre); I. McCarlie and L.M. Campbell (Southbank Surgery, Kirkintilloch); P.H. Edwards and T. Thompson (Ely Bridge Surgery, Cardiff); D.E. Ward (Whitchurch Road, Cardiff); P.A. Corris (Freeman Hospital, Newcastle-upon-Tyne); J.P. Nankani (Hillcrest Medical Centre, Wrexham); A.W. Matthews (Queen Alexandra Hospital, Portsmouth); C.H.C. Twort (St. Thomas' Hospital, London); P.D.O. Davies (Sefton General Hospital, Liverpool); I. Nelemans (St. Albans Medical Centre, Bournemouth); J. Howard (Town End, Caterham); U.B.N. Rau (Horninglow Street, Burton-upon-Trent); S.Y. Sham (Bunbury Road, Birmingham); J.G. Ayres (Birmingham Heartlands Hospital, Birmingham). The results were analysed by E. Beresford and the manuscript prepared by $\mathrm{T}$. Donovan.

\section{References}

1. British Thoracic Society, the British Paediatric Association, the Research Unit of the Royal College of Physicians of London, the King's Fund Centre, the National Asthma Campaign, the Royal College of General Practitioners, 
the General Practitioners in Asthma Group, the British Association of Accident and Emergency Medicine, and the British Paediatric Respiratory Group. Guidelines on the management of asthma. Thorax 1993; 48: S1-S24.

2. Geddes DM. Inhaled corticosteroids: benefits and risks. Thorax 1992; 47: 404-407.

3. Janson-Bjerklie S, Schnell S. Effect of peak flow information on patterns of self-care in adult asthma. Heart Lung 1988; 17: 543-548.

4. Beasley R, Cushley M, Holgate ST. A self-management plan in the treatment of adult asthma. Thorax 1989; 44: 200-204.

5. Charlton I, Charlton G, Broomfield J, Mullee MA.
Evaluation of peak flow and symptoms only self-management plans for the control of asthma in general practice. Br Med J 1990; 301: 1355-1359.

6. Grampian Asthma Study of Integrated Care (GRASSIC). Effectiveness of routine self-monitoring of peak flow in patients with asthma. Br Med $J$ 1994; 308: 564-567.

7. Nunn AJ, Gregg I. New regression equations for predicting peak expiratory flow in adults. $\mathrm{Br} M e d J 1989$; 298: 1068-1070.

8. Corris PA, Hardy CC, Richardson A, Taylor MD, Richardson PDI. The efficacy and acceptability of two breath-actuated, multi-dose, dry-powder inhalers in the treatment of chronic asthma. Br J Clin Res 1992; 3: 139-150. 